\title{
Electronic cigarettes and indoor air quality: a review of studies using human volunteers
}

\begin{abstract}
Objective: This paper is primarily aimed to review articles on electronic cigarettes (ecigarettes) focusing on indoor air quality (IAQ) assessment that were conducted using human volunteers under natural settings that mimic actual vaping scenarios. Such studies may give a better representation of the actual potential exposure towards e-cigarettes emissions in indoor settings. Methods: A systematic literature search was conducted using PubMed search engine database. Search terms such as "electronic cigarette", "e-cigarette", "electronic nicotine delivery system", and "indoor air quality" were used to identify the relevant articles to be included in this review. Articles that involved human volunteers who were asked to vape in natural settings or settings that mimic the actual vaping scenario were chosen to be reviewed. The search yielded a total of 15 published articles. Eleven articles were excluded due to 1) unavailability of its full-text $(n=1), 2)$ did not involve human volunteers $(n=5)$ and 3$)$ did not involve an IAQ study $(n=5)$. Four articles were critically reviewed in this paper. Results: From the four selected articles, two of the papers focused on the determination of nicotine level released by e-cigarettes whereas the other two covered IAQ parameters namely; particulate matters (PM), propylene glycols, formaldehyde, metals and polycyclic aromatic hydrocarbons (PAHs). Only two of the studies involved determination of biomarkers of exposure. The level of chemical contents released varied between studies. The differences in the brands of e-cigarette used, number of vapers recruited and the sensitivity of the methodologies employed in these studies may be the possible causes for such differences. However, studies using human volunteers conducted in a natural setting are more relevant to portray the actual exposure to vapors among e-cigarettes users and non-users compared to studies using a smoking machine/an exposure chamber. This is because such studies take into account the behavior of consumers and individual retention of nicotine. Such method will therefore avoid the possibility of overestimation in terms of exposures toward e-cigarettes users and non-users. Conclusion: There are limited e-cigarette studies on the impact of IAQ performed using human volunteers in natural settings. The available studies however, provided inconsistent scientific evidence on the actual exposure towards the vapor contents as unstandardized methodology were used in conducting such research. Therefore, there is a need to conduct IAQ studies in natural settings by using a standardized protocol in terms of the number of vapers recruited, the size of the indoor settings, the methods used in detecting and quantifying the contents and levels of emissions and the sensitivity of the equipment used in analyzing the contents. This will help in better utilization of the findings from such studies for the use of risk assessment of the exposures towards e-cigarette emissions. There is also a need to emphasize that it is the onus of the manufacturers in providing and proving scientifically sound safety claims for their products prior to commercializing it in the market.
\end{abstract}

Keyword: E-cigarettes; Exhaled aerosol; Indoor air quality; Nicotine; Vaping 